\title{
CLOCKS AND INSENSITIVITY TO SMALL MEASUREMENT ERRORS*
}

\author{
EDUARDo D. SONTAG ${ }^{1}$
}

\begin{abstract}
This paper deals with the problem of stabilizing a system in the presence of small measurement errors. It is known that, for general stabilizable systems, there may be no possible memoryless state feedback which is robust with respect to such errors. In contrast, a precise result is given here, showing that, if a (continuous-time, finite-dimensional) system is stabilizable in any way whatsoever (even by means of a dynamic, time varying, discontinuous, feedback) then it can also be semiglobally and practically stabilized in a way which is insensitive to small measurement errors, by means of a hybrid strategy based on the idea of sampling at a "slow enough" rate.
\end{abstract}

Résumé. Cet article traite du problème de la stabilisation d'un système en présence de petites erreurs de mesure. Il est connu que, pour des systèmes stabilisables généraux, il ne peut pas exister de feedback d'état sans mémoire qui soit résistant à de telles erreurs. À l'opposé, un résultat précis est donné ici, montrant que, si un système (en temps continu et dimension finie) est stabilisable d'une quelconque façon (même à l'aide d'un feedback dynamique, instationnaire, discontinu), alors il peut être semiglobalement et pratiquement stabilisé d'une façon insensible à de petites erreurs de mesure, à l'aide d'une stratégie hybride reposant sur l'idée d'une prise de mesure de l'état à un rythme suffisamment lent.

AMS Subject Classification. 93B52, 93D15.

Received February 26, 1999. Revised September 4, 1999.

\section{INTRODUCTION}

We deal in this paper with control systems

$$
\dot{x}=f(x, u)
$$

for which states $x(t)$ evolve in $\mathcal{X}=\mathbb{R}^{n}$ and controls take values $u(t)$ in a metric space $\mathcal{U}$. The mapping $f: \mathcal{X} \times \mathcal{U} \rightarrow \mathbb{R}^{n}:(x, u) \mapsto f(x, u)$ is continuous, and is locally Lipschitz on $x$ uniformly on compact subsets of $\mathcal{X} \times \mathcal{U}$; additional precise definitions are given later. We suppose given some compact invariant set $\mathcal{A}$ to which we would like to asymptotically drive all states. A special case is that in which $\mathcal{A}=\{0\}$. Our results are new for that particular case, but we state and prove them in more generality, since the proofs are not substantially harder for the general case (such as a periodic trajectory, see Fig. 1).

Keywords and phrases: Control systems, feedback stabilization, controllability.

* Supported in part by US Air Force Grant F49620-98-1-0242.

${ }^{1}$ Department of Mathematics, Rutgers University, New Brunswick, NJ 08903, U.S.A.; e-mail: sontag@hilbert.rutgers.edu 


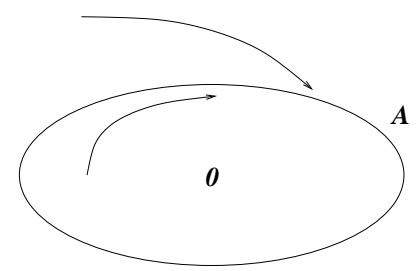

FiguRE 1. Controlling to set.

\section{Stabilization under measurement noise}

The stabilization problem is usually described as that of finding a feedback law $k: \mathcal{X} \rightarrow \mathcal{U}$ such that $\mathcal{A}$ is a globally asymptotically stable set with respect to the closed-loop dynamics

$$
\dot{x}=f(x, k(x)) \text {. }
$$

In this paper, we will be interested in fact in a somewhat stronger notion of stabilization, in which measurement noise may occur. That is, the controller measures $x(t)+e(t)$ at time $t$, where $e(t)$ is an "observation error" and one wants that all trajectories converge to $\mathcal{A}$ provided that the errors e $(t)$ be sufficiently small. The precise value of $e(t)$ is unknown by the controller, but one uses information about upper bounds on the magnitude of $e(t)$ when carrying out the design.

The meaning of "small" will be made precise later, but it is clear that only small errors can be tolerated when close to $\mathcal{A}$. To illustrate, take $\mathcal{A}=\{0\}$ and a stabilizable linear system $\dot{x}=A x+B u$. Suppose that the linear feedback $k(x)=-F x$ stabilizes and $x^{\prime} P x$ is a quadratic Lyapunov function associated to the closed loop system. That is, $P$ is a positive definite symmetric matrix satisfying

$$
(A-B F)^{\prime} P+P(A-B F)=-I .
$$

Writing $d:=-B F e$, we have that $\dot{x}=(A-B F) x+d$ so

$$
\frac{d\left(x^{\prime} P x\right)}{d t}=-|x|^{2}+2 x^{\prime} P d \leq-\frac{|x|^{2}}{2}
$$

whenever $|d| \leq|x| /(4\|P\|)$. This means that stability holds as long as $|e(t)| \leq|x(t)| /(4\|P\|\|B F\|)$ for all $t$. That is, the "margin of measurement error" such that decrease of the Lyapunov function is insured is small when near the set $\mathcal{A}$ (the origin, in this case).

More generally, for arbitrary nonlinear systems, this insensitivity, or robustness, to small errors is also automatic, provided that the feedback law $k$ is a continuous function. To see this, we may consider the perturbed system

$$
\dot{x}=f(x, k(x+e))
$$

which arises when the control $u(t)=k(x(t)+e(t))$ is used instead of $u(t)=k(x(t))$. If $k$ was a stabilizing continuous feedback, then, since $f(x, k(x+e)) \approx f(x, k(x))$ when $e$ is small, the movement of the trajectories is still in the correct direction. This can be proved rigorously in several manners, for instance using Lyapunov functions. Indeed, suppose that $V$ is a Lyapunov function for verifying the asymptotic stability of $\mathcal{A}$ with respect to the closed-loop system $\dot{x}=f(x, k(x))$ (see e.g. [7] together with converse Lyapunov theorems for set stability, such as [10]); thus, $\nabla V(x) \cdot f(x, k(x))<0$ for all $x \notin \mathcal{A}$. Since

$$
\nabla V(x) \cdot f(x, k(x+e)) \approx \nabla V(x) \cdot f(x, k(x)),
$$

we also have that $\nabla V(x) \cdot f(x, k(x+e))<0$ for all $x \in \mathcal{X} \backslash \mathcal{A}$ and all $e(\cdot)$ which satisfy that $|e(t)|<\gamma\left(|x(t)|_{\mathcal{A}}\right)$ for all $t$, where $\gamma: \mathbb{R}_{>0} \rightarrow \mathbb{R}_{>0}$ is some continuous function which can be interpreted as a robustness margin 
for this problem. But this proof breaks down when $k$ is not continuous, because the vector $f(x, k(x+e))$ may point in a very different direction than $f(x, k(x))$.

\section{Discontinuous feedbacks and chattering}

Unfortunately, discontinuous feedback laws $k$ are often unavoidable. For example, for the system $\dot{x}=u$ with $\mathcal{X}=\mathcal{U}=\mathbb{R}$, the set $\mathcal{A}=\{-1,1\}$ can be made globally asymptotically stable under many different discontinuous feedback laws (such as: $k(x)=1-x$ for $x \geq 0$ and $k(x)=-1-x$ for $x<0$ ) but there is no possible continuous global feedback stabilizer $k(x)$ (because the domain of attraction of $\mathcal{A}$ has two connected components).

Even if $\mathcal{A}=\{0\}$, it may be impossible to stabilize continuously. Let us explain this with an intuitive example. We consider the following system $(c f .[2])$ :

$$
\dot{x}=g(x) u, \text { where } g(x)=\left(\begin{array}{c}
x_{1}^{2}-x_{2}^{2} \\
2 x_{1} x_{2}
\end{array}\right) .
$$

The state space is $X=\mathbb{R}^{2}$, and the input space is $\mathcal{U}=\mathbb{R}$ (if we think of points in $\mathbb{R}^{2}$ as complex numbers $z$, this can be described more simply as $\dot{z}=z^{2} u$ ). The orbits of the vector field $g$ are the circles tangent to the $x_{1}$ axis and centered on the $x_{2}$-axis, as well as $\{0\}$ and the positive and negative $x_{1}$ axes, $c f$. Figure 2 . On the circles, input values $u(t)$ that are positive, negative, or zero correspond respectively to motions along the integral curves of $g$ that are counterclockwise, clockwise, or staying at one point, respectively.
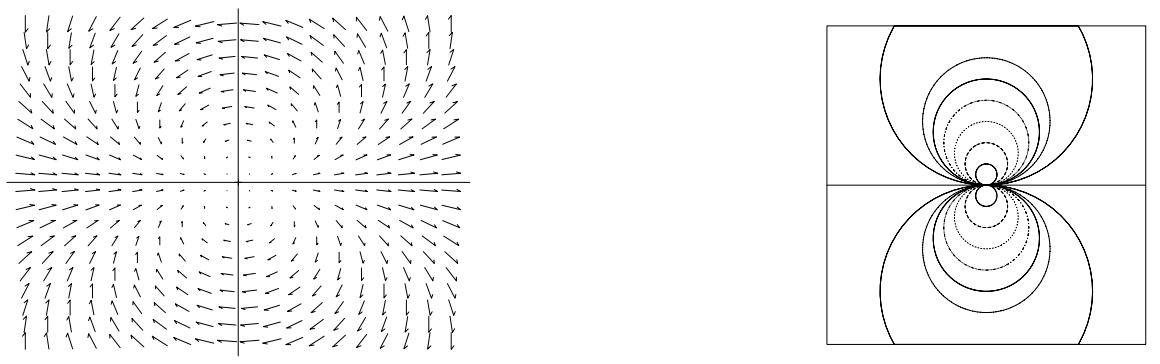

FiguRE 2. $\left(x_{1}^{2}-x_{2}^{2}\right) \frac{\partial}{\partial x_{1}}+2 x_{1} x_{2} \frac{\partial}{\partial x_{2}}$ with typical integral manifolds.

We want to asymptotically stabilize the origin. Since no motion can exit the integral curves, we will simply study what happens in any fixed circle, say one on the top half-plane. (We may think of the system restricted to our circle as a system on $\mathbb{S}^{1}$, with, in polar coordinates, $\dot{\theta}=u$, that is, controls allow us to move at any speed, clockwise or counterclockwise.) Let us call $p$ the bottom point in the circle (the point $0 \in \mathbb{R}^{2}$ ). Near $p$, and to its right, stability says that $u=k(x)$ must make us move clockwise, that is, $k(x)<0$, while near but to the left of $p$ we must move counterclockwise, i.e. $k(x)>0$. If $k$ were to be a continuous function of the state $x$, the intermediate value theorem would force the existence, on this circle, of some state $q \neq p$ at which $k(q)=0$. Thus $q$ is another equilibrium point, and so $k$ cannot asymptotically stabilize $p$. So a continuous feedback stabilizer cannot exist, which means that the argument given earlier for insensitivity to measurement errors cannot be applied.

Of course, there are many obvious discontinuous feedback stabilizers. The most obvious solution is to go clockwise if $x_{1}>0$ and counterclockwise if $x_{1}<0$, making an arbitrary choice, let us say clockwise, if $x_{1}=0$. When restricting to some fixed circle as above, this would mean that one moves each state to the right of the "North" point $p^{\prime}$, including $p^{\prime}$ itself, clockwise towards $p$, and every state to the left of $p^{\prime}$ counterclockwise towards $p$, see Figure 3. However, such feedback laws are extremely sensitive to measurement errors, and may result in chattering behavior at the discontinuity $p^{\prime}$. Indeed, if the true state $x$ is slightly to the left of the top point $p^{\prime}$, but our measurements make us believe it to be to the right, we will mistakenly use a clockwise motion, in effect bringing the state towards the top, instead of downwards towards the target (the origin); see Figure 4 . 


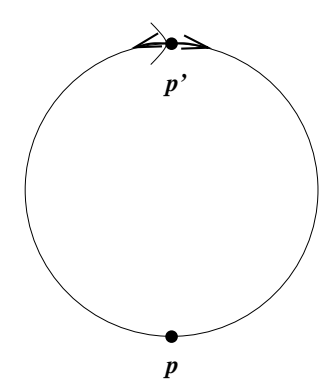

Figure 3. Discontinuous feedback law for circle example.

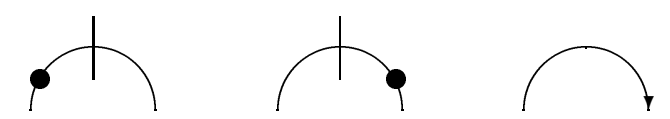

Figure 4. True state; measured state; erroneous motion commanded.

It is clear that, if we are unlucky enough to consistently make measurement errors that place us on the opposite side of the true state, the result may well be an oscillation (chattering) around the top. So the feedback law which we gave is not "robust" with respect to measurement errors, in that it may get "stuck" due to such errors. (We are being deliberately vague, in this intuitive discussion, regarding the meaning of "solution" of the differential equation (3), a nontrivial issue when $k$ is not continuous. This is a very important technical matter, which is discussed in detail in this context in the expository paper [14].)

\section{Necessity of "hybrid" strategies}

It is possible to prove, in a precise technical sense, that, in general, there is no possible memory-free state feedback $u=k(x)$, no matter how discontinuous, which stabilizes in a manner insensitive to measurement errors; see [9]. This negative result is in turn related to previous work by Hermes, Hájek, Coron and Rosier, Ryan et al., and in essence shows that the existence of such feedbacks would imply classical necessary conditions for continuous stabilizability.

Faced with the impossibility of using pure state feedback (if the objective is to stabilize with some robustness to small errors), one may ask about dynamic feedback. There are many techniques common in engineering that are used in order to avoid chattering, such as the introduction of deadzones, which can be understood as dynamic (i.e., memory-based) feedback. In this paper, more specifically, we use an approach based on the idea of "staying the course" required by the controller for a certain minimal time (which depends on assumed upper bounds on the observation error), and to only re-estimate the state, and the resulting value of the feedback law, after this minimal time period has elapsed. In general, one may interpret such a a controller as a "hybrid" one, in the sense that both discrete and continuous time are involved, and logical decisions are taken.

Indeed, in the paper [8], a general theorem is presented, which shows that, if a nonlinear system is at all stabilizable, then it is possible to modify the control law in such a manner that it becomes robust to small errors. The controller given there incorporates an internal model of system. It compares, at appropriate sampling times, the state predicted by the internal model with the -noisy- observations of the state; whenever these differ substantially, a "reseting" is performed on the state of the controller. The result given in the present paper can be seen as an alternative version of the same general idea, but it is somewhat simpler and it requires relatively little work beyond that (for stabilization in the absence of observation noise) found in [4]. The technique, which can be applied in great generality, is based upon the following modification of a given control strategy $k$ which might not be robust to measurement errors. Starting at some initial time, let us say $t_{0}=0$, we measure $k(x(0)+e(0))$, obtaining a control value $u_{0}$. We now apply the constant control $u(t) \equiv u_{0}$ during a small interval $\left[0, t_{1}\right)$. This procedure is then repeated: at time $t_{1}$ we resample, obtaining some $u_{1}:=k\left(x\left(t_{1}\right)+e\left(t_{1}\right)\right)$, and apply this control during another small interval $\left[t_{1}, t_{2}\right)$, and so on. This 
modified strategy is not a pure "continuous time" one, in that a minimum intersample time is required. It is also not a standard sample-and-hold strategy, since the sampling rate is not constant.

Using still the simple example of $\dot{\theta}=u$ in the circle as an illustration, this modified strategy would mean that we decide in which direction to move (clockwise or counterclockwise), based on the current measurement (which may well be somewhat inaccurate). Now, we continue moving in the chosen direction, not paying any attention to observations, for a small time interval. Only after that interval do we again sample. Suppose that our original observation $k(x(0)+e(0))$ tells us that we appear to be near the top point, $p^{\prime}$. Depending on an assumed upper bound on the magnitude of the measurement errors, we can estimate an interval around $p^{\prime}$ where the true state lives. Now we move away and towards the lower point for a certain minimal time whose length depends on this interval. We end up sufficiently far from the discontinuity. At this point, we know for sure on which side of the top we are. Actually, it really doesn't matter if we moved clockwise or counterclockwise; in either case, we will be far enough from $p^{\prime}$ to be sure that errors in the next measurement $k\left(x\left(t_{1}\right)+e\left(t_{1}\right)\right)$ do not lead to catastrophic consequences.

This example is too simple to exhibit the complexity of the general result, especially because there is only one discontinuity, at $p^{\prime}$, in the feedback law, and we are safe once that we are far from $p^{\prime}$ (and, since motions directed by $k$ are in the direction away from $p^{\prime}$, we never go back towards it, if we started far enough away from it). However, it illustrates the general principle that the length of time that we wait must be in some sense proportional to the uncertainty. Actually, the general case is not too difficult to understand. We now explain it intuitively.

\section{The role of Lyapunov functions}

We discussed already the fact that the stability of the unperturbed system (2) implies that of (3) when $k$ is continuous. As a matter of fact, the same implication is true in the more general situation in which there is some continuously differentiable Lyapunov function for the closed-loop system. Basically, this follows from the approximation

$$
\nabla V(x) \cdot f(x, k(x+e)) \approx \nabla V(x+e) \cdot f(x+e, k(x+e))<0
$$

using the Lyapunov property at the point $x+e$ instead of at $x$, and not using continuity of $k$. The proof is given in [9] - see also [14] - where it is also shown that, in general, differentiable Lyapunov functions do not exist. Observe that for our circle example, this alternative argument cannot be applied; the obvious "Lyapunov function" given by the geodesic distance to $p$ measured along the circle is not a differentiable function, since it is nondifferentiable at the conjugate point $p^{\prime}$. However, continuous (but nondifferentiable) Lyapunov functions do play a role.

The general sampling strategy, adapted to a continuous Lyapunov function $V$, is as follows. Suppose that the true current state, let us say at sampling time $t=t_{i}$, is $x$, but that the controller uses $u=k(\tilde{x})$, where $\tilde{x}=x+e$, and $e$ is small. Call $x^{\prime}$ the state that results at the next sampling time $t=t_{i+1}$. By continuity of solutions on initial conditions (recall that the vector fields defining the system are assumed to be continuous), $\left|x^{\prime}-\tilde{x}^{\prime}\right|$ is also small, where $\tilde{x}^{\prime}$ is the state that would have resulted from applying the control $u$ if the true state had been $\tilde{x}$. By continuity, it follows that $V(x) \approx V(\tilde{x})$ and also $V\left(x^{\prime}\right) \approx V\left(\tilde{x}^{\prime}\right)$. On the other hand, a Lyapunov function for the feedback law $k$ has the property that $V\left(\tilde{x}^{\prime}\right)$ is much smaller than $V(\tilde{x})$ if $t_{i+1}-t_{i}$ is large; more precisely, we will have that $V\left(\tilde{x}^{\prime}\right)<V(\tilde{x})-d\left(t_{i+1}-t_{i}\right)$, where $d$ is some positive constant while sufficiently far from $\mathcal{A}$. Hence, if $e$ is sufficiently small compared to the intersample time $t_{i+1}-t_{i}$, it will necessarily be the case that $V\left(x^{\prime}\right)$ must also be smaller than $V(x)$. See Figure 5. Thinking of $V$ as a Lyapunov function, this means that $x^{\prime}$ is made "smaller", even if the wrong control $u=k(\tilde{x})$, rather than $k(x)$, is applied.

\section{Definitions And StATEMEnts of Results}

We now turn to the precise definitions and statements of results. We take $\mathcal{U}$ to be a locally compact metric space, with a distinguished element which we write as " 0 " and denote $|u|:=d(u, 0)$ and, for each $r>0$, 


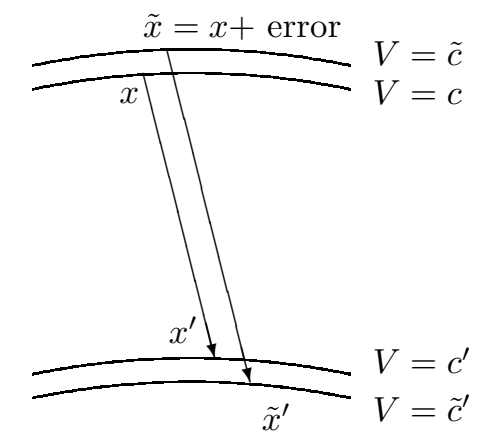

FIGURE 5. $t_{i+1} \gg t_{i} \Rightarrow \tilde{c}^{\prime} \ll \tilde{c} \Rightarrow c^{\prime}<c$.

$\mathcal{U}_{r}:=\{u|| u \mid \leq r\}$. Without loss of generality, we assume that the set $\mathcal{A}$ is invariant when controls are set to zero: all solutions $x(t)$ of the autonomous system $\dot{x}=f(x, 0)$ for which $x(0) \in \mathcal{A}$ have the property that $x(t) \in \mathcal{A}$ for all $t \geq 0$. The mapping $f: \mathcal{X} \times \mathcal{U} \rightarrow \mathbb{R}^{n}:(x, u) \mapsto f(x, u)$ is continuous, and is locally Lipschitz on $x$ uniformly on compact subsets of $\mathcal{X} \times \mathcal{U}$. A control is a measurable essentially locally bounded function $u:[0, \infty) \rightarrow \mathcal{U}$, and $\|u\|$ is the essential supremum (possibly infinite) of the map $t \mapsto|u(t)|$. We denote by $|\xi|_{\mathcal{A}}$ the distance from the point $\xi \in \mathcal{X}$ to the set $\mathcal{A}$ and by $|x|=|x|_{\{0\}}$ the usual Euclidean norm of $x \in X$.

Recall that $\alpha: \mathbb{R}_{\geq 0} \rightarrow \mathbb{R}_{\geq 0}$ is called a $\mathcal{K}_{\infty}$ function if it is continuous, strictly increasing, unbounded, and satisfies $\alpha(0)=0$, and the class of $\mathcal{K} \mathcal{L}$ functions consists of those $\beta: \mathbb{R}_{\geq 0} \times \mathbb{R}_{\geq 0} \rightarrow \mathbb{R}_{\geq 0}$ with the properties that $(1) \beta(\cdot, t) \in \mathcal{K}_{\infty}$ for all $t$, and $(2) \beta(r, t)$ decreases to zero as $t \rightarrow \infty$.

We say that (1) is (globally) asymptotically controllable to the compact and zero-invariant set $\mathcal{A}$ if there exist a $\mathcal{K} \mathcal{L}$-function $\beta$ and a continuous and increasing $\sigma: \mathbb{R}_{\geq 0} \rightarrow \mathbb{R}_{>0}$ such that, for each state $x^{0} \in \mathcal{X}$, there exists a control with $\|u\| \leq \sigma\left(\left|x^{0}\right|_{\mathcal{A}}\right)$, such that the solution $x(t)=x\left(t, x^{0}, u\right)$ of $\dot{x}=f(x, u)$ with $x(0)=x^{0}$ exists for all $t \geq 0$ and satisfies

$$
|x(t)|_{\mathcal{A}} \leq \beta\left(\left|x^{0}\right|_{\mathcal{A}}, t\right) \quad \text { for all } t \geq 0
$$

This bound encapsulates both the stability and attraction properties of the the invariant set $\mathcal{A}$; the role of $\sigma$ is simply to rule out unbounded controls as we approach it. (See e.g. [14] for the equivalence of this definition to the usual topological one.)

By a sampling schedule or partition $\pi=\left\{t_{i}\right\}_{i \geq 0}$ of $[0,+\infty)$ we mean an infinite sequence

$$
0=t_{0}<t_{1}<t_{2}<\ldots
$$

with $\lim _{i \rightarrow \infty} t_{i}=\infty$; its upper and lower diameters are defined by:

$$
\overline{\mathbf{d}}(\pi):=\sup _{i \geq 0}\left(t_{i+1}-t_{i}\right)
$$

and

$$
\underline{\mathbf{d}}(\pi):=\inf _{i \geq 0}\left(t_{i+1}-t_{i}\right) .
$$

By a feedback law for (1) we mean a map $k: \mathcal{X} \rightarrow \mathcal{U}$, with $k \equiv 0$ on the set $\mathcal{A}$, which is locally bounded, that is, the closure of $k(C)$ is compact, for each compact subset $C$ of $\mathcal{X}$. If $k$ is a feedback law, $x^{0} \in \mathcal{X}, \pi$ is a sampling schedule, and $e:[0, \infty) \rightarrow X$ is any function, to be thought of as the state estimation error, we define the $\pi$-solution of

$$
\dot{x}=f(x, k(x+e)), \quad x(0)=x^{0}
$$


as the continuous (and piecewise differentiable) function obtained by recursively solving

$$
\dot{x}(t)=f\left(x(t), k\left(x\left(t_{i}\right)+e\left(t_{i}\right)\right)\right), t \in\left[t_{i}, t_{i+1}\right)
$$

with $x(0)=x^{0}$; that is, for each interval $\left[t_{i}, t_{i+1}\right.$ ), the initial state is measured (with error), the control value $u_{i}=k\left(x\left(t_{i}\right)+e\left(t_{i}\right)\right)$ is computed, and the constant control $u \equiv u_{i}$ is applied until time $t_{i+1}$. The solution is defined on some maximal nontrivial interval; it may fail to exist on the entire interval $[0,+\infty)$ due to a blow-up on one of the subintervals $\left[t_{i}, t_{i+1}\right)$. We denote this maximal solution as $x_{\pi}\left(t, x^{0}, e\right)$ in order to exhibit the dependence on the initial state, $\pi$, and measurement error, and we say that $x_{\pi}\left(t, x^{0}, e\right)$ is well defined if it is defined on all of $[0,+\infty)$.

We now state our main result, a theorem assuring semiglobal practical stability (i.e., driving all states in a given compact set of initial conditions into a specified neighborhood of zero). Note that the error $e$ is assumed to be bounded in sup, rather than "essential sup" norm.

Theorem 2.1. Suppose that the system (1) is globally asymptotically controllable to $\mathcal{A}$. Then, there exists a function $\Gamma \in \mathcal{K}_{\infty}$ with the following property. For each pair $0<\varepsilon<K$, there is a feedback law $k$, and there exist positive $\delta=\delta(\varepsilon, K), \kappa=\kappa(\varepsilon, K)$, and $T=T(\varepsilon, K)$, such that, for each sampling schedule $\pi$ with $\overline{\mathbf{d}}(\pi) \leq \delta$, each $e:[0, \infty) \rightarrow X$ so that

$$
|e(t)| \leq \kappa \underline{\mathbf{d}}(\pi) \quad \forall t \geq 0
$$

and each $x^{0}$ with $\left|x^{0}\right|_{\mathcal{A}} \leq K$, the $\pi$-solution $x(t)=x_{\pi}\left(t, x^{0}, e\right)$ of the noisy system (5) is well-defined and satisfies

$$
|x(t)|_{\mathcal{A}} \leq \Gamma(K) \quad \forall t \geq 0
$$

and

$$
|x(t)|_{\mathcal{A}} \leq \varepsilon \quad \forall t \geq T
$$

This theorem is a generalization of the one given in the paper [4] for systems with no measurement error; actually, the main result in that paper is no more than the particular case $e \equiv 0$ (and $\mathcal{A}=\{0\}$ ). Moreover, our proof is closely patterned after that in [4], and it will show that the same feedback laws, with no modification whatsoever, work also for the more general Theorem 2.1. To be precise, a global feedback in [4] was defined by patching together certain semiglobal feedback laws, denoted there as $k_{\nu}$ and indexed by triples $\nu=(\alpha, r, R)$ of positive numbers with $r<R$; in the proof of our theorem, we will pick $k=k_{\nu}$, for a suitably chosen triple $\nu$ which depends on the given $K$ and $\varepsilon$. Although our proofs, in general terms, proceed along lines parallel to [4], we need to rework all the details because the estimates are a somewhat different when proving the stronger result. In addition, the estimates have to be reworked in order to deal with set stability as opposed to equilibria. The results presented here were outlined in [14], which also described ideas of how to obtain, in a similar manner, a version of Theorem 2.1 in which the same feedback law is picked in different shells. Finally, we remark that a variant of Theorem 2.1 is given in [5], which already pointed out, in the context of discontinuous feedback, the importance of relating measurement error size to step size and of using "nearly uniform" partitions.

One may think of the result in [4] as a solution of a continuous-time stabilization problem. If the system (1) is globally asymptotically controllable to the origin, then there is a feedback $k$ so that the closed-loop system (2) has $x=0$ as a globally asymptotically stable equilibrium. The interpretation of "solution" for the closedloop system equations (2), and therefore of what "globally asymptotically stable equilibrium" really means, is delicate, since when $k$ is not continuous, solutions in the usual sense (absolutely continuous functions whose derivatives satisfy the equation almost everywhere) may not exist for arbitrary initial conditions, and one must therefore first introduce an appropriate generalization of the usual notion. Techniques such as Filippov solutions are not appropriate, $c f$. [6] and [11]. This subject is discussed at length in [4], see also the expository paper [14], and we omit the details here, save to point out that generalized solutions can be interpreted as the limit points 
in the space of continuous paths, as $\overline{\mathbf{d}}(\pi) \rightarrow 0$, of solutions $x_{\pi}$. The "do not sample too fast" results in this paper are qualitatively different, and cannot really be thought of as dealing with continuous-time feedback.

\section{Proofs}

In order to keep the presentation self-contained, we review the necessary concepts of proximal analysis and control-Lyapunov functions, borrowing freely from [4] and other references.

\subsection{Control-Lyapunov functions}

We say that a continuous function

$$
V: X \rightarrow \mathbb{R}_{\geq 0}
$$

is positive definite with respect to $\mathcal{A}$ if $V(x)=0$ if and only if $x \in \mathcal{A}$, and it is proper (or "weakly coercive") if for each $a \geq 0$ the set $\{x \mid V(x) \leq a\}$ is compact, or, equivalently, $V(x) \rightarrow \infty$ as $|x| \rightarrow \infty$ (radial unboundedness). Since $\mathcal{A}$ is compact, the conjunction of the properties of properness and positive definiteness with respect to $\mathcal{A}$ is equivalent to the existence of two functions $\underline{\alpha}, \bar{\alpha} \in \mathcal{K}_{\infty}$ so that

$$
\underline{\alpha}\left(|x|_{\mathcal{A}}\right) \leq V(x) \leq \bar{\alpha}\left(|x|_{\mathcal{A}}\right) \quad \forall x \in \mathcal{X} .
$$

For any continuous $V: X \rightarrow \mathbb{R}$ (or even, just lower semicontinuous and with extended real values), one defines a proximal subgradient of $V$ at the point $x \in \mathcal{X}$ as any vector $\zeta \in \mathbb{R}^{n}$ such that, for some $\theta>0$ and some neighborhood $\mathcal{O}$ of $x$,

$$
V(y) \geq V(x)+\zeta \cdot(y-x)-\theta|y-x|^{2} \quad \forall y \in \mathcal{O} .
$$

In other words, proximal subgradients are the possible gradients of supporting quadratics at the point $x$. The set of all proximal subgradients at $x$ is denoted $\partial_{\mathrm{P}} V(x)$.

A continuous $V: X \rightarrow \mathbb{R}_{\geq 0}$ is a control-Lyapunov function (clf) for the system (1) with respect to the set $\mathcal{A}$ if it is proper, positive definite with respect to $\mathcal{A}$, and infinitesimally decreasing in the following sense: there exist a continuous $W: X \rightarrow \mathbb{R}_{\geq 0}$, positive definite with respect to $\mathcal{A}$, and a nondecreasing $\sigma: \mathbb{R}_{\geq 0} \rightarrow \mathbb{R}_{\geq 0}$, so that

$$
\sup _{x \in X} \max _{\zeta \in \partial_{\mathrm{P}} V(x)} \min _{|u| \leq \sigma\left(|x|_{\mathcal{A}}\right)} \zeta \cdot f(x, u)+W(x) \leq 0 .
$$

For differentiable $V,(7)$ amounts to asking that $\nabla V(x) \cdot f(x, u)<0$ for each $x \notin \mathcal{A}$, by appropriate choices of input values $u=u_{x}$ (with $u_{x}$ uniformly bounded on each compact).

Proposition 3.1. The system (1) is asymptotically controllable if and only if it admits a clf.

Proof. This result is a simple reinterpretation of the one given in [12] for $\mathcal{A}=\{0\}$ and generalized in [1] to arbitrary $\mathcal{A}$. Before quoting these previous results, we recall the notion of relaxed control. A relaxed $\mathcal{U}_{s}-$ valued control is a measurable map $\omega: I \rightarrow \mathbb{P}\left(\mathcal{U}_{s}\right)$, where $I$ is an interval containing zero and $\mathbb{P}\left(\mathcal{U}_{s}\right)$ denotes the set of all Borel probability measures on $\mathcal{U}_{s}$. Note that ordinary controls can be seen also as relaxed controls, via the natural embedding of $\mathfrak{U}_{s}$ into $\mathbb{P}\left(\mathcal{U}_{s}\right)$ (map any point $u \in \mathcal{U}_{s}$ into the Dirac delta measure supported at $u$ ). Given any $\mu \in \mathbb{P}\left(\mathcal{U}_{s}\right)$, we write $\int_{\mathcal{U}_{s}} f(x, u) d \mu(u)$ simply as $f(x, \mu)$. As with ordinary controls, we also denote by $x\left(t, x^{0}, \omega\right)$ the solution of the initial value problem that obtains from initial state $x^{0}$ and relaxed control $\omega$, and we consider the supremum norm $\|\omega\|$, defined as the infimum of the set of $s$ such that $\omega(t) \in \mathbb{P}\left(\mathcal{U}_{s}\right)$ for almost all $t \in I$.

The main result given in [12] for $\mathcal{A}=\{0\}$ and generalized in [1] to arbitrary $\mathcal{A}$ says that if (and only if) a system is $\mathrm{AC}$, there exist two continuous functions $V, W: X \rightarrow \mathbb{R}$, positive definite with respect to $\mathcal{A}$ and with 
$V$ proper, and a nondecreasing function $\sigma: \mathbb{R}_{\geq 0} \rightarrow \mathbb{R}_{\geq 0}$, so that the following property holds: for each $x^{0} \in X$ there are a $T>0$ and a relaxed control $\omega:[0, T) \rightarrow \mathbb{P}\left(\mathcal{U}_{\sigma\left(\left|x^{0}\right|_{\mathcal{A}}\right)}\right)$, so that $x(t):=x\left(t, x^{0}, \omega\right)$ is defined for all $0 \leq t<T$ and

$$
V(x(t))-V\left(x^{0}\right) \leq-\int_{0}^{t} W(x(\tau)) d \tau \quad \forall t \in[0, T) .
$$

To simplify notations, let us write $s:=\sigma\left(\left|x^{0}\right|_{\mathcal{A}}\right)$. In order to obtain the proximal version of the result, we show that

$$
\min _{|u| \leq s} \zeta \cdot f\left(x^{0}, u\right) \leq-W\left(x^{0}\right)
$$

for all $\zeta \in \partial_{\mathrm{P}} V\left(x^{0}\right)$ (which may well be empty).

As in [15], we first make an intermediate reduction, showing that

$$
\min _{v \in F\left(x^{0}, s\right)} D V\left(x^{0} ; v\right) \leq-W\left(x^{0}\right),
$$

where $F\left(x^{0}, s\right)$ denotes the (closed) convex hull of $\left\{f\left(x^{0}, u\right), u \in \mathcal{U}_{s}\right\}$, and $D V\left(x^{0} ; v\right)$ is the directional subderivate, or contingent epiderivative, of $V$ in the direction of $v$ at $x^{0}$, defined as

$$
D V\left(x^{0} ; v\right):=\liminf _{\substack{t \searrow 0 \\ v^{\prime} \rightarrow v}} \frac{1}{t}\left[V\left(x^{0}+t v^{\prime}\right)-V\left(x^{0}\right)\right] .
$$

(The minimum in (10) is achieved, because the map $v \mapsto D V\left(x^{0} ; v\right)$ is lower semicontinuous, see e.g. [3], Ex. 3.4.1e.) To prove $(10)$, let $x(t)=x\left(t, x^{0}, \omega\right)$ be as above, and consider for each $t \in[0, T)$ the vectors

$$
r_{t}:=\frac{1}{t}\left(x(t)-x^{0}\right)=\frac{1}{t} \int_{0}^{t} f(x(\tau), \omega(\tau)) d \tau=q_{t}+p_{t},
$$

with

$$
p_{t}:=\frac{1}{t} \int_{0}^{t} f\left(x^{0}, \omega(\tau)\right) d \tau,
$$

where $q_{t} \rightarrow 0$ as $t \searrow 0$ (the existence of such a $q_{t}$, for each $t$, is an easy consequence of the fact that $f$ is locally Lipschitz on $x$, uniformly on $u \in \mathcal{U}_{s}$ ). Moreover, $p_{t} \in F\left(x^{0}, s\right)$ for all $t$ (because $F\left(x^{0}, s\right)$ is convex, so $f\left(x^{0}, \omega(\tau)\right) \in F\left(x^{0}, s\right)$ for each $\tau$, and then using convexity once more). By compactness, we have that there is some $v \in F\left(x^{0}, s\right)$ and some subsequence $p_{t_{j}} \rightarrow v$ with $t_{j} \backslash 0$; as $q_{t} \rightarrow 0$, also $v_{j}:=r_{t_{j}} \rightarrow v$. For this $v$,

$$
D V\left(x^{0} ; v\right) \leq \liminf _{j \rightarrow \infty} \frac{1}{t_{j}}\left[V\left(x^{0}+t_{j} v_{j}\right)-V\left(x^{0}\right)\right]=\liminf _{j \rightarrow \infty} \frac{1}{t_{j}}\left[V\left(x\left(t_{j}\right)\right)-V\left(x^{0}\right)\right] \leq-W\left(x^{0}\right)
$$

(using (8)), so (10) indeed holds.

Finally, we show that (10) implies (9). Let $v \in F\left(x^{0}, s\right)$ achieve the minimum, and pick any $\zeta \in \partial_{\mathrm{P}} V\left(x^{0}\right)$. By definition of proximal subgradient, there is some $\theta>0$ so that, for each $v^{\prime} \in F\left(x^{0}, s\right)$ and each small $t \geq 0$,

$$
\zeta \cdot v^{\prime} \leq \frac{1}{t}\left[V\left(x^{0}+t v^{\prime}\right)-V\left(x^{0}\right)\right]+\theta t\left|v^{\prime}\right|^{2}
$$

so taking limits along any sequence $t \backslash 0$ and $v^{\prime} \rightarrow v$ shows that $\zeta \cdot v \leq D V\left(x^{0} ; v\right) \leq-W\left(x^{0}\right)$. By definition of $F\left(x^{0}, s\right)$, this means that there must exist a $u \in \mathcal{U}_{s}$ so that also $\zeta \cdot f\left(x^{0}, u\right) \leq-W\left(x^{0}\right)$, as desired. 
Observe that the proximal condition (9), being linear on the velocities $f(x, u)$, has the great advantage of not requiring convex hulls in order to state, and in that sense is far more elegant than (10).

From now on, we assume given a clf $V$ for the system (1), and $\underline{\alpha}$ and $\bar{\alpha}$ are $\mathcal{K}_{\infty}$ functions as in (6). We define

$$
\rho(s):=\bar{\alpha}^{-1}\left(\frac{1}{2} \underline{\alpha}(s)\right)
$$

for each $s \geq 0$.

\subsection{Regularization and definition of feedback}

For each $\alpha \in(0,1]$ and $x \in \mathcal{X}$, we pick one point $y_{\alpha}(x) \in \mathcal{X}$ such that

$$
V\left(y_{\alpha}(x)\right)+\frac{1}{2 \alpha^{2}}\left|y_{\alpha}(x)-x\right|^{2} \leq V(y)+\frac{1}{2 \alpha^{2}}|y-x|^{2}
$$

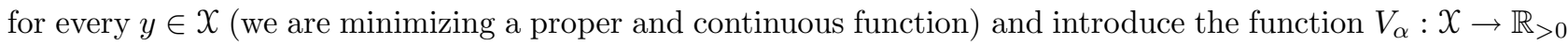
(Iosida-Moreau regularization) defined by

$$
V_{\alpha}(x):=V\left(y_{\alpha}(x)\right)+\frac{1}{2 \alpha^{2}}\left|y_{\alpha}(x)-x\right|^{2} .
$$

Note that (use $y=x$ in (12)) for each $x$,

$$
V_{\alpha}(x) \leq V(x) .
$$

For each $x, \alpha$, and the choice made of $y_{\alpha}(x)$, we define the vector

$$
\zeta_{\alpha}(x):=\frac{x-y_{\alpha}(x)}{\alpha^{2}}
$$

and rearrange terms in (12) to obtain, for any $y \in \mathcal{X}$,

$$
V(y) \geq V\left(y_{\alpha}(x)\right)+\zeta_{\alpha}(x) \cdot\left(y-y_{\alpha}(x)\right)-\frac{1}{2 \alpha^{2}}\left|y-y_{\alpha}(x)\right|^{2},
$$

which implies, by definition of subgradients,

$$
\zeta_{\alpha}(x) \in \partial_{\mathrm{P}} V\left(y_{\alpha}(x)\right) .
$$

Note that (13) and (14) imply

$$
\frac{1}{2 \alpha^{2}}\left|y_{\alpha}(x)-x\right|^{2} \leq V(x)-V\left(y_{\alpha}(x)\right) \leq V(x),
$$

so, denoting

$$
B_{R}^{\mathcal{A}}:=\left\{\left.x|| x\right|_{\mathcal{A}} \leq R\right\}
$$

and recalling that $V(x) \leq \bar{\alpha}\left(|x|_{\mathcal{A}}\right)$,

$$
\left|y_{\alpha}(x)-x\right| \leq \sqrt{2 \bar{\alpha}(R)} \alpha \quad \forall x \in B_{R}^{\mathcal{A}} .
$$


In general, one has $|x+y|_{\mathcal{A}} \leq|x|_{\mathcal{A}}+|y|$ for any two $x, y \in \mathcal{X}$, so we have

$$
x \in B_{R}^{\mathcal{A}} \Rightarrow y_{\alpha}(x) \in \mathcal{B}_{R}:=B_{R+\sqrt{2 \bar{\alpha}(R)}}^{\mathcal{A}}
$$

(using that $\alpha \leq 1$ ).

Next, we associate as in [4] a feedback law $k=k_{\nu}$ defined on $B_{R}^{\mathcal{A}}$ to each triple $\nu=(\alpha, r, R)$ with $0<r<R$ and $\alpha \in(0,1]$. With the function $\sigma$ as in equation (7), we let

$$
\widetilde{\mathcal{U}}_{R}:=\{u \in \mathcal{U}|| u \mid \leq \sigma(R+\sqrt{2 \bar{\alpha}(R)})\} .
$$

Finally, we define $k_{\nu}: B_{R}^{\mathcal{A}} \rightarrow \widetilde{\mathcal{U}}_{R}$ by letting $k_{\nu}(x)$ be any pointwise minimizer of $\zeta_{\alpha}(x) \cdot f(x, u)$ :

$$
\zeta_{\alpha}(x) \cdot f\left(x, k_{\nu}(x)\right)=\min _{u \in \widetilde{\mathcal{U}}_{R}} \zeta_{\alpha}(x) \cdot f(x, u) \text {. }
$$

(Actually, $k$ does not depend on $r$, but merely on $R$ and $\alpha$, but we keep the notation in [4].) If $|x|_{\mathcal{A}} \leq R$, then (7) applied at $x=y_{\alpha}(x)$ and $\zeta=\zeta_{\alpha}(x)$, and in view of (20), gives

$$
\min _{u \in \widetilde{\mathcal{U}}_{R}} \zeta_{\alpha}(x) \cdot f\left(y_{\alpha}(x), u\right) \leq-W\left(y_{\alpha}(x)\right) .
$$

Proposition 3.2. Pick any $0<r<R$ so that $2 \underline{\alpha}(r)<\underline{\alpha}(R)$. Then there exist positive numbers $\alpha, \delta, \kappa, T$ such that, for each sampling schedule $\pi$ with $\overline{\mathbf{d}}(\pi) \leq \bar{\delta}$, each $\bar{e}:[0, \infty) \rightarrow X$ so that

$$
|e(t)| \leq \kappa \underline{\mathbf{d}}(\pi) \quad \forall t \geq 0,
$$

and each $x^{0}$ with $\left|x^{0}\right|_{\mathcal{A}} \leq \frac{1}{2} \rho(R)$, the $\pi$-solution $x(t)=x_{\pi}\left(t, x^{0}, e\right)$ of the noisy system (5) obtained when using the feedback $k=k_{\alpha, r, R}$ is well-defined and satisfies

$$
x(t) \in B_{R}^{\mathcal{A}} \quad \forall t \geq 0
$$

and

$$
x(t) \in B_{r}^{\mathcal{A}} \quad \forall t \geq T .
$$

Theorem 2.1 is an immediate corollary of this proposition. Indeed, we may pick the $\mathcal{K}_{\infty}$-function $\Gamma:=(\rho / 2)^{-1}$ :

$$
\Gamma(s):=\underline{\alpha}^{-1}(2 \bar{\alpha}(2 s)) .
$$

Then, given $\varepsilon$ and $K$ as in the statement of Theorem 2.1, we let $R:=\Gamma(K)$ (so, $K=(1 / 2) \rho(R)$ ), and pick any $r>0$ such that $r<\varepsilon$ and $2 \underline{\alpha}(r) \leq \underline{\alpha}(R)$; applying the result to $0<r<R$ gives the desired conclusion.

We prove Proposition 3.2 through a series of technical steps. Let $0<r<R$ be given, with $2 \underline{\alpha}(r)<\underline{\alpha}(R)$. The set $\widetilde{U}_{R}$ is as in (21).

Some continuity estimates

We need some additional notations:

$$
\begin{aligned}
m_{R} & :=\max _{x \in B_{R}^{\mathcal{A}}, u \in \widetilde{\mathcal{U}}_{R}}|f(x, u)|, \\
\Delta & :=\frac{1}{3} \min \left\{W(y) \mid y \in \mathcal{B}_{R} \text { and } r \leq \Gamma\left(|y|_{\mathcal{A}}\right)\right\}
\end{aligned}
$$


(from (11) and (26), the last condition says that $\frac{1}{2} \rho(r) \leq|y|_{\mathcal{A}}$ ),

$$
|f(x, u)-f(y, u)| \leq \ell|x-y| \quad \forall u \in \widetilde{\mathcal{U}}_{R}, \forall x, y \in \mathcal{B}_{R},
$$

(Lipschitz constant for $f$ with respect to $x$, uniformly on $u \in \widetilde{\mathcal{U}}_{R}$ ), and

$$
\omega_{R}(\delta):=\max \left\{V(x)-V(y)|| x-y \mid \leq \delta, x, y \in \mathcal{B}_{R}\right\}
$$

(modulus of continuity of $V$ on $\mathcal{B}_{R}$ ). Of course, $\omega_{R}(\delta) \rightarrow 0$ as $\delta \rightarrow 0$.

For any $\alpha \in(0,1]$, we consider the function $V_{\alpha}$. By definition, we have

$$
V_{\alpha}(y) \leq V\left(y_{\alpha}(x)\right)+\frac{1}{2 \alpha^{2}}\left|y_{\alpha}(x)-y\right|^{2}
$$

and

$$
V_{\alpha}(x)=V\left(y_{\alpha}(x)\right)+\frac{1}{2 \alpha^{2}}\left|y_{\alpha}(x)-x\right|^{2}
$$

for all $x, y$, so subtracting and rearranging quadratic terms, we obtain:

$$
V_{\alpha}(y) \leq V_{\alpha}(x)+\zeta_{\alpha}(x) \cdot(y-x)+\frac{1}{2 \alpha^{2}}|y-x|^{2} .
$$

On the other hand (15) and (19) give that

$$
\left|\zeta_{\alpha}(x)\right| \leq \frac{\sqrt{2 \bar{\alpha}(R)}}{\alpha}
$$

so from (30) it follows that

$$
V_{\alpha}(y)-V_{\alpha}(x) \leq c|y-x|
$$

where

$$
c:=\frac{\sqrt{2 \bar{\alpha}(R)}}{\alpha}+\frac{R+D}{\alpha^{2}}
$$

and $D:=\max _{a, b \in \mathcal{A}}|a-b|$ is the diameter of $\mathcal{A}$. Interchanging $x$ and $y$, we conclude that $c$ is a Lipschitz constant for $V_{\alpha}$ on the set $B_{R}^{\mathcal{A}}$. Without loss of generality (using if required a smaller $\alpha$ in the arguments to follow) we will assume $c \geq 2$.

We also have the following estimate, for any $x \in B_{R}^{\mathcal{A}}$ :

$$
V(x) \leq V_{\alpha}(x)+\omega_{R}(\sqrt{2 \bar{\alpha}(R)} \alpha) .
$$

This is true because $V(x) \leq V\left(y_{\alpha}(x)\right)+\omega_{R}\left(\left|y_{\alpha}(x)-x\right|\right), V\left(y_{\alpha}(x)\right) \leq V_{\alpha}(x)$, and (19).

Choice of $\alpha$ and feedback

Finally, we pick any $\alpha>0$ which satisfies all the following conditions:

$$
\omega_{R}(\sqrt{2 \bar{\alpha}(R)} \alpha)<\frac{1}{2} \underline{\alpha}(R),
$$




$$
\sqrt{2 \bar{\alpha}(R)} \alpha<\frac{1}{2} \rho(r), \quad 2 \ell \omega_{R}(\sqrt{2 \bar{\alpha}(R)} \alpha)<\Delta
$$

and

$$
\omega_{R}(\sqrt{2 \bar{\alpha}(R)} \alpha)<\frac{1}{16} \underline{\alpha}(r)
$$

and take the feedback $k=k_{\alpha, r, R}$.

\subsection{The main technical lemma}

Note that, for any $s>0,|x|_{\mathcal{A}} \leq \rho(s)$ implies $V(x) \leq \bar{\alpha}(\rho(s))=\frac{1}{2} \underline{\alpha}(s)$. Define, for any $s$,

$$
G_{s}:=\left\{x \mid V(x) \leq \frac{1}{2} \underline{\alpha}(s)\right\}
$$

and

$$
G_{s}^{\alpha}:=\left\{x \mid V_{\alpha}(x) \leq \frac{1}{2} \underline{\alpha}(s)\right\}
$$

The first two inclusions that follow are then clear, using (14):

$$
B_{\rho(R)}^{\mathcal{A}} \subseteq G_{R} \subseteq G_{R}^{\alpha} \subseteq \operatorname{int} B_{R}^{\mathcal{A}}
$$

The last inclusion is true because $V_{\alpha}(x) \leq \frac{1}{2} \underline{\alpha}(R)$ implies, by (29),

$$
V\left(y_{\alpha}(x)\right) \leq \frac{1}{2} \underline{\alpha}(R) \text { and } \frac{1}{2 \alpha^{2}}\left|y_{\alpha}(x)-x\right|^{2} \leq \frac{1}{2} \underline{\alpha}(R),
$$

and therefore $y_{\alpha}(x) \in B_{R}^{\mathcal{A}}$ and $\left|y_{\alpha}(x)-x\right| \leq \sqrt{\underline{\alpha}(R)} \alpha<\sqrt{2 \bar{\alpha}(R)} \alpha$, from which we get

$$
V(x) \leq V\left(y_{\alpha}(x)\right)+\omega_{R}(\sqrt{2 \bar{\alpha}(R)} \alpha)<\underline{\alpha}(R),
$$

which in turn implies that $|x|_{\mathcal{A}}<R$ by definition of $\underline{\alpha}(R)$.

Next, we pick any $\varepsilon_{0}>0$ so that all the following - somewhat redundant - properties hold, where $B$ denotes the unit ball and $B_{R}^{\mathcal{A}}$ is as defined in (18):

$$
\begin{gathered}
B_{\frac{1}{2} \rho(R)}^{\mathcal{A}}+\varepsilon_{0} B \subseteq G_{R}^{\alpha}, \\
G_{R}^{\alpha}+2 \varepsilon_{0} B \subseteq B_{R}^{\mathcal{A}}, \\
\varepsilon_{0} \leq \frac{\underline{\alpha}(r)}{8 c} \leq \frac{\underline{\alpha}(r)}{16}, \\
\underline{\alpha}(r)+c \varepsilon_{0}<\frac{1}{2} \underline{\alpha}(R)
\end{gathered}
$$

(recall that $2 \underline{\alpha}(r)<\underline{\alpha}(R)$ ), and

$$
G_{r}^{\alpha}+2 \varepsilon_{0} B \subseteq G_{R}^{\alpha}
$$


(If $x \in \operatorname{int} B_{R}^{\mathcal{A}}$ then for any $\eta$ such that $x+\eta \in B_{R}^{\mathcal{A}}$ we have, since $c$ is a Lipschitz constant for $V_{\alpha}$ on $B_{R}^{\mathcal{A}}$, that $V_{\alpha}(x+\eta) \leq V_{\alpha}(x)+c|\eta|$, and thus, for any $x$ so that $V_{\alpha}(x) \leq \frac{1}{2} \underline{\alpha}(r) \leq \frac{1}{4} \underline{\alpha}(R)$ it holds that $V_{\alpha}(x+\eta) \leq \frac{1}{2} \underline{\alpha}(R)$ for $\eta$ small.)

We let $\delta_{0}$ be any positive number so that, for every initial state $x^{0}$ in the compact set $G_{R}^{\alpha}$, and for each control $u:\left[0, \delta_{0}\right] \rightarrow \widetilde{\mathcal{U}}_{R}$, the solution of $\dot{x}=f(x, u)$ with $x(0)=x^{0}$ is defined on the entire interval $\left[0, \delta_{0}\right]$ and satisfies $x(t) \in G_{R}^{\alpha}+\varepsilon_{0} B$ for all $t$.

Finally, we pick any $\delta>0$ which satisfies:

$$
\begin{gathered}
\left(\frac{\ell m \sqrt{2 \bar{\alpha}(R)}}{\alpha}+\frac{m^{2}}{2 \alpha^{2}}\right) \delta \leq \Delta . \\
\omega_{R}(m \delta)<\frac{1}{4} \underline{\alpha}(r),
\end{gathered}
$$

and

$$
\delta \leq \min \left\{1, \delta_{0}, \frac{2}{\Delta} \varepsilon_{0}, \frac{\underline{\alpha}(r)}{8 c m}\right\}
$$

We take, for the proof of Proposition 3.2:

$$
\kappa:=\frac{\Delta}{4 c} e^{-\ell}
$$

and

$$
T:=\frac{\underline{\alpha}(R)}{\Delta}
$$

The main technical fact needed is as follows.

Lemma 3.3. Let $0<r<R$ satisfy $2 \underline{\alpha}(r)<\underline{\alpha}(R)$. Let $\alpha, \delta, \kappa, T$ be defined as above, and $k$ be the feedback $k_{\alpha, r, R}$. Pick any $\varepsilon>0$, and consider the following set:

$$
P=P_{r, R, \varepsilon}:=\left\{x \mid x+\varepsilon B \subseteq G_{R}^{\alpha}\right\} .
$$

Let $\pi$ be a partition which satisfies

$$
\frac{\varepsilon}{\kappa} \leq t_{i+1}-t_{i} \leq \delta \quad \forall i=0,1, \ldots
$$

(that is, $\overline{\mathbf{d}}(\pi) \leq \delta$ and $\varepsilon \leq \kappa \underline{\mathbf{d}}(\pi)$ ). Then, for any $e:[0, \infty) \rightarrow X$ such that $|e(t)| \leq \varepsilon$ for all $t$, and any $x^{0} \in P$, the $\pi$-solution $x(t)=x_{\pi}\left(t, x^{0}, e\right)$ of the noisy system (5) obtained when using the feedback $k=k_{\alpha, r, R}$ is well-defined and satisfies (24) and (25), and $x\left(t_{i}\right) \in P$ for all $i$.

We will prove this via a couple of lemmas, but let us first point out how Proposition 3.2 follows from Lemma 3.3. Suppose given a partition $\pi$ with $\overline{\mathbf{d}}(\pi) \leq \delta$, an error function $e$ so that $|e(t)| \leq \kappa \underline{\mathbf{d}}(\pi)$ for all $t$, and an initial state $x^{0}$ with $\left|x^{0}\right|_{\mathcal{A}} \leq \frac{1}{2} \rho(R)$. All we need to establish is that $x^{0} \in P$, so that the Lemma applies with this initial condition. Let $\varepsilon:=\sup _{t \geq 0}|e(t)| \leq \kappa \underline{\mathbf{d}}(\pi)$. Note that

$$
\varepsilon \leq \kappa \underline{\mathbf{d}}(\pi) \leq \kappa \overline{\mathbf{d}}(\pi) \leq \kappa \delta \leq \kappa \frac{2}{\Delta} \varepsilon_{0} \leq \kappa \frac{4 c}{\Delta} e^{\ell} \varepsilon_{0}=\varepsilon_{0} .
$$


Thus, by (36),

$$
B_{\frac{1}{2} \rho(R)}^{\mathcal{A}} \subseteq P,
$$

so $x^{0} \in P$.

\subsection{Proof of Lemma 3.3}

Observe that (46) implies $\varepsilon \leq \kappa \underline{\mathbf{d}}(\pi)$, so, arguing as in (47), $\varepsilon \leq \varepsilon_{0}$. It is useful to introduce the following set as well:

$$
Q=Q_{r, R, \varepsilon}:=G_{r}^{\alpha}+\varepsilon B
$$

Observe that $Q \subseteq P$, by (40).

We start the proof by establishing an analogue of Lemma IV.2 in [4]. This is perhaps the critical step, in so far as it introduces a trade-off between the "contraction" due to controller action and the "expansion" forced by measurement errors.

Lemma 3.4. If, for some index $i, x_{i}:=x\left(t_{i}\right) \in P \backslash Q$, then $x(t)$ is defined for all $t \in\left[t_{i}, t_{i+1}\right]$,

$$
\begin{gathered}
x(t) \in B_{R}^{\mathcal{A}} \quad \forall t \in\left[t_{i}, t_{i+1}\right], \\
V_{\alpha}(x(t)) \leq V_{\alpha}\left(x_{i}\right)+\varepsilon_{0} \quad \forall t \in\left[t_{i}, t_{i+1}\right],
\end{gathered}
$$

and, letting $x_{i+1}:=x\left(t_{i+1}\right)$ :

$$
\begin{gathered}
x_{i+1} \in P \\
V_{\alpha}\left(x_{i+1}\right)-V_{\alpha}\left(x_{i}\right) \leq-\frac{\Delta}{2}\left(t_{i+1}-t_{i}\right) .
\end{gathered}
$$

Proof. By definition of $P$, we have that $\tilde{x}_{i}:=x_{i}+e\left(t_{i}\right) \in G_{R}^{\alpha}$. Also, $\tilde{x}_{i} \notin G_{r}^{\alpha}$, since otherwise $x_{i}$ would belong to $Q$. In particular, $\tilde{x}_{i} \notin B_{\rho(r)}^{\mathcal{A}}$. Let $\tilde{x}(\cdot)$ be the solution, on $\left[t_{i}, t_{i+1}\right]$, of $\dot{x}=f\left(x, k\left(\tilde{x}_{i}\right)\right)$ with $\tilde{x}\left(t_{i}\right)=\tilde{x}_{i}$. It follows from Lemma A.3 that this solution is well-defined, $\tilde{x}\left(t_{i+1}\right) \in G_{R}^{\alpha}$, and

$$
V_{\alpha}(\tilde{x}(t))-V_{\alpha}\left(\tilde{x}_{i}\right) \leq-\Delta\left(t-t_{i}\right)
$$

for all $t \in\left[t_{i}, t_{i+1}\right)$.

As $x_{i} \in P \subseteq G_{R}^{\alpha}$, and $t_{i+1}-t_{i} \leq \overline{\mathbf{d}}(\pi) \leq \delta \leq \delta_{0}$, the definition of $\delta_{0}$ insures that the solution $x(\cdot)$ of $\dot{x}=f\left(x, k\left(\tilde{x}_{i}\right)\right)$ with $x\left(t_{i}\right)=x_{i}$ is also well-defined and remains in $G_{R}^{\alpha}+\varepsilon_{0} B \subseteq B_{R}^{\mathcal{A}}$ for all $t \in\left[t_{i}, t_{i+1}\right)$. So, by Gronwall's inequality, we know that

$$
|x(t)-\tilde{x}(t)| \leq e^{\left(t-t_{i}\right) \ell}\left|x_{i}-\tilde{x}_{i}\right| \leq e^{\delta \ell} \varepsilon
$$

for all $t \in\left[t_{i}, t_{i+1}\right]$. Since $V_{\alpha}$ has Lipschitz constant $c$ on $B_{R}^{\mathcal{A}}$, we have

$$
\begin{aligned}
V_{\alpha}(x(t))-V_{\alpha}\left(x_{i}\right)= & V_{\alpha}(x(t))-V_{\alpha}(\tilde{x}(t))+V_{\alpha}(\tilde{x}(t))-V_{\alpha}\left(\tilde{x}_{i}\right)+V_{\alpha}\left(\tilde{x}_{i}\right)-V_{\alpha}\left(x_{i}\right) \\
& \leq c e^{\delta \ell} \varepsilon-\Delta\left(t-t_{i}\right)+c \varepsilon \leq \frac{\Delta}{2}\left(t_{i+1}-t_{i}\right)-\Delta\left(t-t_{i}\right)
\end{aligned}
$$


for all $t \in\left[t_{i}, t_{i+1}\right]$, where we have used that $\delta \leq 1, \varepsilon \leq \kappa \underline{\mathbf{d}}(\pi)$, the definition $\kappa=(\Delta / 4 c) e^{-\ell}$, and the fact that $\underline{\mathbf{d}}(\pi) \leq t_{i+1}-t_{i}$. In particular, the estimate (52) results at $t=t_{i+1}$, and (50) holds because $(\Delta / 2) \delta \leq \varepsilon_{0}$ by $(43)$.

We are only left to prove that $x_{i+1} \in P$. By definition of $P$, this means that for any given $\eta \in \mathcal{X}$ with $|\eta| \leq \varepsilon$ it must hold that

$$
\eta+x_{i+1} \in G_{R}^{\alpha}=\left\{x \mid V_{\alpha}(x) \leq \frac{1}{2} \underline{\alpha}(R)\right\} .
$$

Pick such an $\eta$; then $\left|\eta+x_{i+1}-\tilde{x}\left(t_{i+1}\right)\right| \leq \varepsilon+e^{\delta \ell} \varepsilon \leq 2 e^{\ell} \varepsilon$, and so, since $\eta+x_{i+1} \in G_{R}^{\alpha}+2 \varepsilon_{0} B \subseteq B_{R}^{\mathcal{A}}$,

$$
V_{\alpha}\left(\eta+x_{i+1}\right) \leq 2 c e^{\ell} \varepsilon+V_{\alpha}\left(\tilde{x}\left(t_{i+1}\right)\right) \leq 2 c e^{\ell} \varepsilon+V_{\alpha}\left(\tilde{x}_{i}\right)-\Delta\left(t_{i+1}-t_{i}\right) \leq V_{\alpha}\left(\tilde{x}_{i}\right)-\frac{\Delta}{2}\left(t_{i+1}-t_{i}\right) \leq \frac{1}{2} \underline{\alpha}(R)
$$

where we again used $\varepsilon \leq \kappa \underline{\mathbf{d}}(\pi)$ as well as the fact that $V_{\alpha}\left(\tilde{x}_{i}\right) \leq(1 / 2) \underline{\alpha}(R)\left(\right.$ since $\left.\tilde{x}_{i} \in G_{R}^{\alpha}\right)$.

We also need another observation, this one paralleling the proof of Lemma IV.4 in [4].

Lemma 3.5. If, for some index $i, x_{i}:=x\left(t_{i}\right) \in Q$, then $x(t)$ is defined for all $t \in\left[t_{i}, t_{i+1}\right]$,

$$
V_{\alpha}(x(t)) \leq \frac{3}{4} \underline{\alpha}(r) \quad \forall t \in\left[t_{i}, t_{i+1}\right],
$$

and

$$
V(x(t)) \leq \frac{7}{8} \underline{\alpha}(r) \quad \forall t \in\left[t_{i}, t_{i+1}\right] .
$$

In particular, $x(t) \in B_{r}^{\mathcal{A}}$ for all $t \in\left[t_{i}, t_{i+1}\right]$ and $x\left(t_{i+1}\right) \in P$.

Proof. The fact that $x$ is defined follows from the choice of $\delta_{0}$, and we know that $x(t) \in B_{R}^{\mathcal{A}}$ for all $t \in\left[t_{i}, t_{i+1}\right]$. So

$$
\left|x(t)-x_{i}\right| \leq m \delta \quad \forall t \in\left[t_{i}, t_{i+1}\right] .
$$

By definition of $Q$, we may write $x_{i}=x^{\prime}+\eta$, for some $x^{\prime} \in G_{r}^{\alpha}$ and some $|\eta| \leq \varepsilon$. Thus, $V_{\alpha}\left(x_{i}\right) \leq V_{\alpha}\left(x^{\prime}\right)+c \varepsilon_{0} \leq$ $\frac{1}{2} \underline{\alpha}(r)+c \varepsilon_{0}$ (second inequality by definition of $G_{r}^{\alpha}$ ). Together with (56), this gives

$$
V_{\alpha}(x(t)) \leq \frac{1}{2} \underline{\alpha}(r)+c \varepsilon_{0}+c m \delta \leq \frac{3}{4} \underline{\alpha}(r) \quad \forall t \in\left[t_{i}, t_{i+1}\right]
$$

(using (38) and (43)). So, using (34) and (31),

$$
V(x(t)) \leq V_{\alpha}(x(t))+\omega_{R}(\sqrt{2 \bar{\alpha}(R)} \alpha) \leq \frac{3}{4} \underline{\alpha}(r)+\frac{1}{16} \underline{\alpha}(r)<\frac{7}{8} \underline{\alpha}(r)
$$

for all $t \in\left[t_{i}, t_{i+1}\right]$, as wanted. So $x(t) \in B_{r}^{\mathcal{A}}$ for all $t \in\left[t_{i}, t_{i+1}\right]$. Finally, if $|\eta| \leq \varepsilon$ then

$$
V_{\alpha}\left(x\left(t_{i+1}\right)+\eta\right) \leq c \varepsilon+\frac{3}{4} \underline{\alpha}(r) \leq \frac{1}{2} \underline{\alpha}(R)
$$

(the last inequality by (39)), which means that $x\left(t_{i+1}\right)+\eta \in G_{R}^{\alpha}$; this implies that $x\left(t_{i+1}\right) \in P$.

Back to the proof of Lemma 3.3, since $x^{0} \in P$, Lemmas 3.4 and 3.5 guarantee that the solution exists for all $t$ and remains in $B_{R}^{\mathcal{A}}$, and that $x_{i}:=x\left(t_{i}\right) \in P$ for all $i$.

Moreover, if there is some $j$ so that $x\left(t_{j}\right) \in Q$, then it holds that $V_{\alpha}\left(x_{i}\right) \leq \frac{3}{4} \underline{\alpha}(r)$ for all $i>j$. This is because on intervals in which $x_{i-1} \in Q$, we already know that $V_{\alpha}\left(x\left(t_{i}\right)\right) \leq \frac{3}{4} \underline{\alpha}(r)$, and if instead $x_{i-1} \in P \backslash Q$, 
then we have $V_{\alpha}\left(x_{i}\right)<V_{\alpha}\left(x_{i-1}\right)$. So, for any such $i>j$, either $x(t) \in B_{r}^{\mathcal{A}}$ for all $t \in\left[t_{i}, t_{i+1}\right]$ (if $x_{i} \in Q$ ) or $V_{\alpha}(x(t)) \leq \frac{3}{4} \underline{\alpha}(r)+\varepsilon_{0}$ for all $t \in\left[t_{i}, t_{i+1}\right]$ (if $x_{i} \in P \backslash Q$ ). Actually, in this last case we also have

$$
\begin{aligned}
V(x(t)) & \leq V_{\alpha}(x(t))+\omega_{R}(\sqrt{2 \bar{\alpha}(R)} \alpha) \leq \frac{3}{4} \underline{\alpha}(r)+\varepsilon_{0}+\omega_{R}(\sqrt{2 \bar{\alpha}(R)} \alpha) \\
& \leq \frac{3}{4} \underline{\alpha}(r)+\frac{1}{16} \underline{\alpha}(r)+\frac{1}{16} \underline{\alpha}(r)<\underline{\alpha}(r),
\end{aligned}
$$

(using (38) and (34)), so, again $x(t) \in B_{r}^{\mathcal{A}}$ for all $t \in\left[t_{i}, t_{i+1}\right]$. In conclusion, trajectories stay in $B_{r}^{\mathcal{A}}$ after the first time that $x\left(t_{j}\right) \in Q$. So we only need to show that there is such a $j$, with $t_{j} \leq T$.

Suppose instead that for $i=0, \ldots, k$ it holds that $x\left(t_{i}\right) \notin Q$, and $t_{k}>T$. Applying (52) repeatedly,

$$
0 \leq V_{\alpha}\left(x\left(t_{k}\right)\right) \leq V_{\alpha}\left(x^{0}\right)-\frac{\Delta}{2} t_{k}<\frac{\alpha}{2}(R)-\frac{\Delta}{2} T
$$

(recall that $x^{0} \in P$ implies that $x^{0} \in G_{R}^{\alpha}$ ), and this contradicts (45). The proof of Lemma 3.3 is then complete.

\section{Non-Global StABILITY AND SyStems ON MANIFOLDS}

A local version of Theorem 2.1 holds. Suppose that (1) is locally asymptotically controllable to the compact and zero-invariant set $\mathcal{A}$, meaning that there are a compact subset $\mathcal{U}_{0} \subseteq \mathcal{U}$, a constant $\rho>0$, and a $\beta \in \mathcal{K} \mathcal{L}$, so that for each $x^{0}$ with $\left|x^{0}\right|<\rho$ there is some $\mathcal{U}_{0}$-valued control $u$ so that $x\left(t, x^{0}, u\right)$ exists for all $t \geq 0$ and satisfies (4). Then, there are $\rho^{\prime}>0, \Gamma \in \mathcal{K}_{\infty}$, and for each pair $\varepsilon<K$ with $\varepsilon>0$ and $K<\rho^{\prime}$ there are a feedback law $k$, and $\delta, \kappa, T$, such that, whenever $\overline{\mathbf{d}}(\pi) \leq \delta,|e(t)| \leq \kappa \underline{\mathbf{d}}(\pi)$ for all $t \geq 0$, and $\left|x^{0}\right|_{\mathcal{A}} \leq K$, the solution $x_{\pi}\left(t, x^{0}, e\right)$ is well-defined and satisfies $|x(t)|_{\mathcal{A}} \in \Gamma(K)$ for $t \geq 0$ and $|x(t)|_{\mathcal{A}} \leq \varepsilon$ for $t \geq T$. This is a consequence of the global result, as we sketch next.

We first introduce a new system which is globally asymptotically controllable to $\mathcal{A}$ and which reduces to the original system in the neighborhood $B_{\rho / 2}^{\mathcal{A}}$. Such a system can be easily obtained in the form $\dot{x}=f(x, u)+\varphi(x) v$, where $\varphi$ is a smooth function which equals 1 outside $B_{2 \rho / 3}^{\mathcal{A}}$ and is zero on $B_{\rho / 2}^{\mathcal{A}}$, and $v \in \mathbb{R}^{n}$ represents a set of $n$ additional controls. Now we apply the global theorem to this extended system. Finally, we pick $\rho^{\prime}$ small enough so that $\Gamma\left(\rho^{\prime}\right)<\rho / 2$, which insures that when starting in a state with $\left|x^{0}\right|_{\mathcal{A}} \leq \rho^{\prime}$ the trajectories will remain in $B_{\rho / 2}^{\mathcal{A}}$ and hence be trajectories of the original system.

More generally, one may give a version of our main result for systems in which the state space is a general (second countable) differentiable manifold $X$ instead of $\mathbb{R}^{n}$ (one restates the stability definitions in topological terms, independent of a metric). Of course, the meaning of "measurement error" must be clarified, since the sum $x(t)+e(t)$ makes no sense if $\mathcal{X}$ is not a vector space. It should be interpreted as using feedback $u=k(z(t))$, where $z(t)$ is close to $x(t)$ in a suitable metric. We sketch next a possible way to achieve such a generalization.

Given a system on $\mathcal{X}$, which is asymptotically controllable to the compact subset $\mathcal{A}$, we embed $X$ in an Euclidean space $\mathbb{R}^{k}$, and extend the dynamics to $\mathbb{R}^{k}$ in such a manner that (1) when restricted to $\mathcal{X}$ we recover the original dynamics, and (2) the extended system is still asymptotically controllable to $\mathcal{A}$. Now, a feedback law for the extension restricts to one for the original system, and it has the same robustness properties as the extension.

The construction of this extension can proceed as follows. We first embed $X$ as a closed submanifold of $\mathbb{R}^{k}$ (Whitney), and extend the original system to a tubular neighborhood of $\mathcal{X}$. On this tubular neighborhood, we modify the dynamics by adding a term that attracts (contracts) to $X$ in the normal direction (and so is zero on $X$ ). This gives an extension to the tube, preserving controllability. Finally, on a closed set disjoint from $X$ but suitably overlapping the tube, we add a term (as " $+\varphi(x) v$ " above) which allows one to control all states in the embedding space $\mathbb{R}^{k}$ into the tube. We leave the details for future work. 


\section{Appendix A. Some lemmas}

The following is Lemma IV.1 from [4]; it is included here in order to make the paper self-contained.

Lemma A.1. Let $\alpha \in(0,1]$ satisfy (33). Then, for any $x \in B_{R}^{\mathcal{A}} \backslash B_{\rho(r)}^{\mathcal{A}}$ it holds that

$$
\zeta_{\alpha}(x) \cdot f\left(x, k_{\nu}(x)\right) \leq-2 \Delta
$$

Proof. It follows from $(22,27)$, and $(23)$, that

$\zeta_{\alpha}(x) \cdot f\left(x, k_{\nu}(x)\right) \leq \min _{u \in \widetilde{\mathcal{U}}_{R}} \zeta_{\alpha}(x) \cdot f\left(y_{\alpha}(x), u\right)+\ell\left|\zeta_{\alpha}(x)\right|\left|y_{\alpha}(x)-x\right| \leq-W\left(y_{\alpha}(x)\right)+\ell\left|\zeta_{\alpha}(x)\right|\left|y_{\alpha}(x)-x\right|$.

On the other hand, the definitions of $\zeta_{\alpha}(x)$ and $\omega_{R}$, together with equations $(17,19)$, and $(20)$, give

$$
\left|\zeta_{\alpha}(x)\right|\left|y_{\alpha}(x)-x\right| \leq 2\left(V(x)-V\left(y_{\alpha}(x)\right)\right) \leq 2 \omega_{R}(\sqrt{2 \bar{\alpha}(R)} \alpha)
$$

so we can conclude that

$$
\zeta_{\alpha}(x) \cdot f\left(x, k_{\nu}(x)\right) \leq-W\left(y_{\alpha}(x)\right)+2 \ell \omega_{R}(\sqrt{2 \bar{\alpha}(R)} \alpha)
$$

We have that $\left|y_{\alpha}(x)-x\right| \leq \sqrt{2 \bar{\alpha}(R)} \alpha \leq \rho(r) / 2$ by $(19)$ and $(33)$, so $\left|y_{\alpha}(x)\right|_{\mathcal{A}} \geq \frac{1}{2} \rho(r)$. Since also $y_{\alpha}(x) \in \mathcal{B}_{R}$, we have the lower bound $W\left(y_{\alpha}(x)\right) \geq 3 \Delta$, so (57) now follows from (58) and (20).

Actually, it is possible to sharpen the above lemma and show that the (locally Lipschitz) function $V_{\alpha}$ behaves like a clf on the sets

$$
C_{r, R}:=\left\{\left.x \in X|r \leq| x\right|_{\mathcal{A}} \leq R\right\}
$$

for all $\alpha$ small enough. We do not need this result explicitly, but it is worth proving it, since the needed techniques are already introduced. We introduce the modulus of continuity of $W$ on $\mathcal{B}_{R}$,

$$
\widetilde{\omega}_{R}(\delta):=\max \left\{W(x)-W(y)|| x-y \mid \leq \delta, x, y \in \mathcal{B}_{R}\right\}
$$

and write

$$
m_{r, R}:=\frac{1}{3} \min \left\{W(x) \mid x \in C_{r, R}\right\}
$$

Lemma A.2. Let $\alpha \in(0,1]$ satisfy

$$
\max \left\{2 \ell \omega_{R}(\sqrt{2 \bar{\alpha}(R)} \alpha), \widetilde{\omega}_{R}(\sqrt{2 \bar{\alpha}(R)} \alpha)\right\} \leq m_{r, R}
$$

Then, for all $x \in C_{r, R}$ and all $\zeta \in \partial_{\mathrm{P}} V_{\alpha}(x)$,

$$
\min _{u \in \widetilde{\mathcal{U}}_{R}} \zeta \cdot f(x, u) \leq-m_{r, R}
$$

Proof. We start by remarking that, for all $\alpha \in(0,1]$ and all $x \in B_{R}^{\mathcal{A}}$,

$$
\left|y_{\alpha}(x)-x\right|^{2} \leq 2 \alpha^{2} \omega_{R}(\sqrt{2 \bar{\alpha}(R)} \alpha)
$$


From (17) and (19),

$$
\frac{1}{2 \alpha^{2}}\left|y_{\alpha}(x)-x\right|^{2} \leq V(x)-V\left(y_{\alpha}(x)\right) \leq \omega_{R}\left(\left|y_{\alpha}(x)-x\right|\right) .
$$

Thus,

$$
\left|\zeta_{\alpha}(x)\right| \cdot\left|y_{\alpha}(x)-x\right|=\frac{\left|y_{\alpha}(x)-x\right|^{2}}{\alpha^{2}} \leq 2 \omega_{R}(\sqrt{2 \bar{\alpha}(R)} \alpha)
$$

and hence

$$
\zeta_{\alpha}(x) \cdot\left[f\left(y_{\alpha}(x), u\right)-f(x, u)\right] \leq \ell\left|\zeta_{\alpha}(x)\right| \cdot\left|y_{\alpha}(x)-x\right| \leq 2 \ell \omega_{R}(\sqrt{2 \bar{\alpha}(R)} \alpha)
$$

for all $u \in \widetilde{\mathcal{U}}_{R}$ and all $x \in B_{R}^{\mathcal{A}}$, because $y_{\alpha}(x) \in \mathcal{B}_{R}$. Thus,

$$
\zeta_{\alpha}(x) \cdot f(x, u) \leq \zeta_{\alpha}(x) \cdot f\left(y_{\alpha}(x), u\right)+2 \ell \omega_{R}(\sqrt{2 \bar{\alpha}(R)} \alpha) .
$$

As $x \in C_{r, R}$, we have that $y_{\alpha}(x) \in \mathcal{B}_{R}$ and $\zeta_{\alpha}(x) \in \partial_{\mathrm{P}} V\left(y_{\alpha}(x)\right)$, so we can pick a $u_{x} \in \widetilde{\mathcal{U}}_{R}$ so that $\zeta_{\alpha}(x)$. $f\left(y_{\alpha}(x), u_{x}\right) \leq-W\left(y_{\alpha}(x)\right) \leq-W(x)+\widetilde{\omega}_{R}(\sqrt{2 \bar{\alpha}(R)} \alpha) \leq-2 m_{r, R}$. Using now the fact that $2 \ell \omega_{R}(\sqrt{2 \bar{\alpha}(R)} \alpha)$ $\leq m_{r, R}$, we conclude that

$$
\zeta_{\alpha}(x) \cdot f(x, u) \leq-m_{r, R}
$$

So we only need to prove that $\partial_{\mathrm{P}} V_{\alpha}(x) \subseteq\left\{\zeta_{\alpha}(x)\right\}$ for all $x$.

Pick any $\zeta \in \partial_{\mathrm{P}} V_{\alpha}(x)$. By definition, this means that exists some $\theta>0$ such that, for all $y$ near $x$, $\zeta \cdot(y-x) \leq V_{\alpha}(y)-V_{\alpha}(x)+\theta|y-x|^{2}$, so

$$
\zeta \cdot(y-x) \leq V_{\alpha}(y)-V_{\alpha}(x)+\gamma(|y-x|)
$$

for some $\gamma(r)=o(r)$. Adding

$$
-\zeta_{\alpha}(x) \cdot(y-x) \leq-V_{\alpha}(y)+V_{\alpha}(x)+\frac{1}{2 \alpha^{2}}|y-x|^{2},
$$

which holds because of (30), we conclude

$$
\left(\zeta-\zeta_{\alpha}(x)\right) \cdot(y-x) \leq o(|y-x|)
$$

Substituting $y=x+h\left(\zeta-\zeta_{\alpha}(x)\right)$ and letting $h \backslash 0$ shows that $\zeta=\zeta_{\alpha}(x)$.

Observe that we have proved more that claimed: equation (60) is satisfied by any viscosity subgradient $\zeta$; in particular, the gradient of $V$, if $V$ happens to be differentiable at the point $x$, must coincide with $\zeta_{\alpha}(x)$. It is worth noticing that feedback constructed in [4] was $k(x)=$ any $u \in \widetilde{\mathcal{U}}_{R}$ minimizing $\zeta_{\alpha}(x) \cdot f(x, u)$ (where $\alpha$ and $\widetilde{U}_{R}$ are chosen constant on certain compacts). As $V_{\alpha}$ is locally Lipschitz, it is differentiable almost everywhere. Thus, the proof of Lemma A.2 insures that $\zeta_{\alpha}(x)=\nabla V_{\alpha}(x)$ for almost all $x$. So $k(x)=u$ is, at those points, the pointwise minimizer of the Hamiltonian $\nabla V_{\alpha}(x) \cdot f(x, u)$ associated to the regularized clf $V_{\alpha}$.

This is basically Lemma IV.2 in [4]: 
Lemma A.3. Let $\alpha, \delta$ satisfy (32, 33), and (41). Pick any $\tau \leq \delta$. Suppose that $x^{0} \in G_{R}^{\alpha} \backslash B_{\rho(r)}^{\mathcal{A}}$, write $u_{0}=k\left(x^{0}\right)$, and consider the maximal solution $x$ of $\dot{x}(t)=f\left(x(t), u_{0}\right), x(0)=x^{0}, t \in[0, \tau]$. Then, $x$ is defined on all of $[0, \tau]$ and

$$
V_{\alpha}(x(t))-V_{\alpha}\left(x^{0}\right) \leq-\Delta t
$$

holds for all $t \in[0, \tau]$. In particular, $x(t) \in G_{R}^{\alpha}$ for all such $t$.

Proof. By (35), $x^{0} \in \operatorname{int} B_{R}^{\mathcal{A}}$, so there is a largest $\bar{t} \in(0, \tau]$ such that $x(s) \in B_{R}^{\mathcal{A}}$ for all $s \in[0, \bar{t}]$. Now choose an arbitrary $t \in[0, \bar{t}]$. Since $m_{R}$ bounds the values $f\left(x(s), u_{0}\right)$ for $s \in[0, \bar{t}]$,

$$
\left|x(t)-x^{0}\right| \leq m_{R} t
$$

and

$$
x(t)=x^{0}+t v,
$$

where

$$
v:=\frac{1}{t} \int_{0}^{t} f\left(x(s), u_{0}\right) d s .
$$

Let us write $\eta:=v-f\left(x^{0}, u_{0}\right)$. Thus, using the bound on $f$ and its Lipschitz constant $\ell$, we obtain the following estimates:

$$
|v| \leq m_{R}, \quad|\eta| \leq \ell m_{R} t
$$

Equation (30) gives

$$
V_{\alpha}\left(x^{0}+t v\right) \leq V_{\alpha}\left(x^{0}\right)+t \zeta_{\alpha}\left(x^{0}\right) \cdot v+\frac{t^{2}|v|^{2}}{2 \alpha^{2}}
$$

so we conclude that

$$
V_{\alpha}(x(t))-V_{\alpha}\left(x^{0}\right)=V_{\alpha}\left(x^{0}+t v\right)-V_{\alpha}\left(x^{0}\right) \leq t \zeta_{\alpha}\left(x^{0}\right) \cdot v+\frac{1}{2 \alpha^{2}} t^{2}|v|^{2} .
$$

We can use $(19,64)$, and Lemma A.1 in order to estimate:

$$
\zeta_{\alpha}\left(x^{0}\right) \cdot v \leq \zeta_{\alpha}\left(x^{0}\right) \cdot f\left(x^{0}, u_{0}\right)+\left|\zeta_{\alpha}\left(x^{0}\right)\right||\eta| \leq-2 \Delta+\frac{\sqrt{2 \bar{\alpha}(R)}}{\alpha} \ell m_{R} \delta
$$

which implies that

$$
V_{\alpha}(x(t))-V_{\alpha}\left(x^{0}\right) \leq\left(-2 \Delta+\frac{\sqrt{2 \bar{\alpha}(R)}}{\alpha} \ell m_{R} \delta+\frac{1}{2 \alpha^{2}} m_{R}^{2} \delta\right) t .
$$

Using (41), this implies (61) for all $t \in\left(0, \bar{t}\right.$. In particular, (61) implies that $x(\bar{t}) \in \operatorname{int} B_{R}^{\mathcal{A}}$, which contradicts the maximality of $\bar{t}$ unless $\bar{t}=\tau$. Therefore (61) holds for all $t \in[0, \tau)$. 


\section{REFERENCES}

[1] F. Albertini and Sontag E.D., Continuous control-Lyapunov functions for asymptotically controllable time-varying systems, Internat. J. Control. to appear. (See also Control-Lyapunov functions for time-varying set stabilization, Proc. European Control Conf., Brussels, July 1997, Paper ECC515.)

[2] Z. Artstein, Stabilization with relaxed controls. Nonl. Anal. TMA 7 (1983) 1163-1173.

[3] F.H. Clarke, Yu.S. Ledyaev, R.J. Stern and P. Wolenski, Nonsmooth Analysis and Control Theory. Springer-Verlag, New York (1998).

[4] F.H. Clarke, Yu.S. Ledyaev, E.D. Sontag and A.I. Subbotin, Asymptotic controllability implies feedback stabilization. IEEE Trans. Automat. Control 42 (1997) 1394-1407.

[5] F.H. Clarke, Yu.S. Ledyaev, L. Rifford and R. Stern, Feedback stabilization and Lyapunov functions. preprint, Univ. de Lyon (1999).

[6] J.-M. Coron and L. Rosier, A relation between continuous time-varying and discontinuous feedback stabilization. J. Math. Systems, Estimation, and Control 4 (1994) 67-84.

[7] J. Kurzweil, On the inversion of Ljapunov's second theorem on stability of motion. Amer. Math. Society Translations, Series 224 (1956) 19-77.

[8] Yu.S. Ledyaev and E.D. Sontag, A remark on robust stabilization of general asymptotically controllable systems, in Proc. Conf. on Information Sciences and Systems (CISS 97), John Hopkins University, Baltimore (1997) 246-251.

[9] Yu.S. Ledyaev and E.D. Sontag, A Lyapunov characterization of robust stabilization. J. Nonl. Anal. 37 (1999) $813-840$.

[10] Y. Lin, E.D. Sontag and Y. Wang, A smooth converse Lyapunov theorem for robust stability, SIAM J. Control Optim. 34 (1996) 124-160.

[11] E.P. Ryan, On Brockett's condition for smooth stabilizability and its necessity in a context of nonsmooth feedback. SIAM J. Control Optim. 32 (1994) 1597-1604.

[12] E.D. Sontag A Lyapunov-like characterization of asymptotic controllability. SIAM J. Control Optim. 21 (1983) $462-471$.

[13] E.D. Sontag, Mathematical Control Theory, Deterministic Finite Dimensional Systems, Second Edition. Springer-Verlag, New York (1998).

[14] E.D. Sontag, Stability and stabilization: Discontinuities and the effect of disturbances, in Nonlinear Analysis, Differential Equations, and Control, Proc. NATO Advanced Study Institute, Montreal, Jul/Aug 1998; F.H. Clarke and R.J. Stern, Eds., Kluwer, Dordrecht (1999) 551-598. See also Nonlinear Control Abstracts \#NCA-8-2-981026, Oct 1998.

[15] E.D. Sontag and H.J. Sussmann, Nonsmooth Control Lyapunov Functions, in Proc. IEEE Conf. Decision and Control, New Orleans, IEEE Publications (1995) 2799-2805. 